\title{
CONGRUENCES IN UNITARY SPACE
}

\author{
BY \\ N. COBURN
}

1. Introduction. In this paper, we shall study the properties of a congruence of $\infty^{n-1}$ curves which are imbedded in a unitary space of $n$ dimensions $K_{n}$ (a real topological space of $2 n$ dimensions). First, we consider the general case-when the curves are $\infty^{n-1}$ unitary curves $K_{1}$ (real topological spaces of two dimensions) - and determine the associated congruence affinors. Then, we determine the necessary and sufficient conditions in terms of congruence vectors that the $\infty^{n-1}$ congruence curves should be either unitary $U_{1}$ (unitary Euclidean curves) ${ }^{1}$ ) or real curves $X_{1}$ (real topological spaces of one dimension). If the curves of the congruence are all real $X_{1}$, then we define the congruence to be real; if the curves are all unitary $U_{1}$, then we define the congruence to be complex Euclidean.

In the next section, we study two systems of Pfaffians which enable us to define two types of orthogonality: (1) $\infty^{1}$ hypersurfaces which are completely unitary orthogonal to the congruence curves; (2) $\infty^{1}$ hypersurfaces which are semi-unitary orthogonal to the congruence curves. It is shown that: (1) the $\infty^{1}$ hypersurfaces which are completely unitary orthogonal to the congruence curves admit of an intrinsic parameterization and are $\infty^{1}$ unitary $K_{n-1}$; (2) if the $\infty^{1}$ hypersurfaces which are semi-unitary orthogonal to the congruence curves admit of a parameterization, then they constitute $\infty^{1}$ semianalytic $\left({ }^{2}\right)$ spaces $X_{n-1}$. A further analytical characterization of these two types of surfaces is given.

The remainder of our work deals with two problems: (1) a characterization in terms of congruence affinors of those congruences which are either completely unitary orthogonal or semi-unitary orthogonal to $\infty^{1}$ hypersurfaces in $K_{n}$; (2) special properties of these two types of congruences. Thus, in connection with the second problem, it is shown that if the congruence is either real, or complex Euclidean, analytic and completely unitary orthogonal to $\infty^{1}$ hypersurfaces, then the conditions satisfied by the congruence vector are similar to those satisfied by the congruence vector which is orthogonal to $\infty^{1}$ hypersurfaces $\left(^{3}\right)$ in $V_{n}$ ( $n$-dimensional Riemannian space). Again, if : (1) the congruence is real and geodesic; (2) the $K_{n}$ has a symmetric connection, then every two hypersurfaces which are semi-unitary orthogonal to the

Presented to the Society, February 28, 1942; received by the editors February 3, 1942.

(1) $[5$, vol. 2 , p. 251$]$.

(2) $[3$, equation $(2.10)]$.

(3) $[5$, vol. 2 , p. 28 , equation 5.2$]$. 
congruence intercept equal arc segments on all $X_{1}$ of the congruence. This latter result is similar to a theorem $\left(^{4}\right)$ in Riemannian space.

2. Notation $\left({ }^{5}\right)$. Consider a real space of $2 n$ dimensions $X_{2 n}$ whose coordinates are given by the real variables

$$
x^{\lambda}, y^{\lambda}
$$$$
\lambda, \mu=1,2, \cdots, n \text {. }
$$

Into this $X_{2 n}$, we introduce the complex coordinates

$$
\begin{aligned}
\xi^{\lambda} & =x^{\lambda}+i y^{\lambda}, & i=(-1)^{1 / 2}, \\
\xi^{\lambda *} & =x^{\lambda}-i y^{\lambda} . &
\end{aligned}
$$

Since the Jacobian of this transformation $(-2 i)$ does not vanish over $X_{2 n}$, the $\xi^{\lambda}, \xi^{\lambda^{*}}$ constitute a set of $2 n$ independent variables which map the $X_{2 n}$. In view of the fact that $\xi^{\lambda^{*}}$ are complex conjugate to $\xi^{\lambda}$, we can determine the points of $X_{2 n}$ by assigning complex numbers to merely $\xi^{\lambda}$. Hence, we say that the $\xi^{\lambda}$ determine "points" which build a complex space of $n$ dimensions (the above real topological $X_{2 n}$ ).

Let us denote partial derivatives by

$$
\partial_{\mu}=\partial / \partial \xi^{\mu}, \quad \partial_{\mu^{*}}=\partial / \partial \xi^{*} .
$$

If $\psi\left(\xi^{\lambda}, \xi^{\lambda^{*}}\right)$ is an analytic function of $\xi^{\lambda}, \xi^{\lambda^{*}}$, then we shall say that $\psi$ is semianalytic; if $\phi\left(\xi^{\lambda}\right)$ is an analytic function of $\xi^{\lambda}$ (or $\xi^{\lambda^{*}}$ ) alone, then we shall say that $\phi$ is analytic. In view of (2.4), we may express this last condition by

$$
\partial_{\mu} \cdot \phi=0 \text {. }
$$

One further important formal idea must be noted-that of the conjugate function and equation. If we replace $i$ by $-i$ in $\phi$, the resulting function is denoted by $\phi^{*}$ (where $\phi$ is a scalar). From (2.2), (2.3), we see that $\xi^{\lambda}$ must be replaced by $\xi^{\lambda^{*}}$ and vice versa. Hence $\phi\left(\xi^{\lambda}\right)$ becomes $\phi^{*}\left(\xi^{\lambda^{*}}\right)$. In the case of affinors, the conjugate affinor is obtained in the same manner. However, we shall indicate this conjugate by starring the previously unstarred indices and removing the star from the previously starred indices. Thus the conjugate of $v_{\lambda_{\mu} *}$ is $v_{\lambda^{*} \mu}$. Furthermore from our discussion, it follows that

$$
\partial_{\mu} \phi^{*}=0 \text {. }
$$

The equation (2.6) is the so-called conjugate equation to (2.5). Also to every affinor equation, there corresponds a conjugate equation obtained by replacing $i$ by $-i$ and hence each affinor by its conjugate. The truth of this last statement can be seen by decomposing each affinor into its real and imaginary parts $\left.{ }^{6}\right)$. In the following, we shall indicate the validity of the conjugate equation by the abbreviation "conj."

(4) $[5$, vol. 2, p. 31].

(s) Our notation is that of [5].

( ${ }^{\circ}$ Note, by composite differentiation, it follows that $\partial_{\mu}=\partial / \partial x^{\mu}-i \partial / \partial y^{\mu}, \partial_{\mu^{*}}=\partial / \partial x^{\mu}$ $+i \partial / \partial y^{\mu}$. 
We specify that the group of this complex $X_{n}$ shall be the analytic group $\left({ }^{7}\right)$ of coordinate transformations. Now, let us introduce a connection in $X_{n}$. by means of the $n^{3}$ quantities $\Gamma_{\mu \alpha}^{\lambda}$ which are functions of the coordinates $\xi^{\lambda}, \xi^{\lambda^{*}}$. We define the covariant differential of a contravariant vector $v^{\lambda}\left(\xi^{\lambda}, \xi^{\lambda^{*}}\right)$ by

$$
\delta v^{\lambda}=d v^{\lambda}+\Gamma_{\mu \alpha}^{\lambda} v^{\alpha} d \xi^{\mu} \text {, conj. }
$$

Likewise, we define the covariant differential of a covariant vector $w_{\lambda}\left(\xi^{\lambda}, \xi^{\lambda^{*}}\right)$ by

$$
\delta w_{\lambda}=d w_{\lambda}-\Gamma_{\mu \lambda}^{\alpha} w_{\alpha} d \xi^{\mu}, \text { conj. }
$$

By expanding the ordinary differential of a vector, we obtain

$$
\begin{aligned}
d v^{\lambda} & =d \xi^{\mu} \partial_{\mu} v^{\lambda}+d \xi^{*} \partial_{\mu^{*} \cdot v^{\lambda}, \text { conj., }} \\
d w_{\lambda} & =d \xi^{\mu} \partial_{\mu} w_{\lambda}+d \xi^{*} \partial_{\mu} \cdot w_{\lambda}, \text { conj.. }
\end{aligned}
$$

If we define the covariant derivative of $v^{\lambda}, w_{\lambda}$ by the equations

$$
\begin{array}{rlrl}
\nabla_{\mu} v^{\lambda} & =\partial_{\mu}^{*} v^{\lambda}+\Gamma_{\mu \alpha}^{\lambda} v^{\alpha}, & \nabla_{\mu} w_{\lambda}=\partial_{\mu} w_{\lambda}-\Gamma_{\mu \lambda}^{\alpha} w_{\alpha}, \text { conj., } \\
\nabla_{\mu} v^{\lambda}=\partial_{\mu} v^{\lambda}, & \nabla_{\mu} \cdot w_{\lambda}=\partial_{\mu} \cdot w_{\lambda}, \text { conj., }
\end{array}
$$

then by use of the equations (2.9) through (2.12), the equations (2.7), (2.8) become

$$
\begin{aligned}
\delta v^{\lambda} & =d \xi^{\mu} \nabla_{\mu} v^{\lambda}+d \xi^{\mu^{*}} \nabla_{\mu} \cdot v^{\lambda}, \text { conj., } \\
\delta w_{\lambda} & =d \xi^{\mu} \nabla_{\mu} w_{\lambda}+d \xi^{\mu *} \nabla_{\mu} * w_{\lambda}, \text { conj. }
\end{aligned}
$$

An hermitian $X_{n}$ with covariant derivative defined by (2.11), (2.12) is denoted by $K_{n}$.

Let us introduce an hermitian tensor with hermitian symmetry, that is,

$$
a_{\lambda \mu^{*}}=\left[\left(a_{\lambda \mu} \cdot\right)^{*}\right]^{\prime}=a_{\mu^{*} \lambda},
$$

the sign (') indicating the transpose matrix. If we condition the $a_{\lambda \mu}$. by requiring that

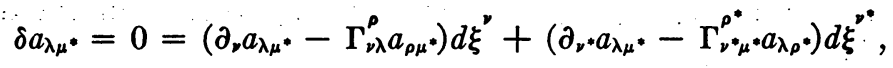

then the space $K_{n}$ is said to be a unitary $K_{n}$. For such a space from (2.16), we can show $\left({ }^{8}\right)$

$$
\begin{aligned}
& \nabla_{\nu} a_{\lambda \mu^{*}}=\partial_{\nu} a_{\lambda_{\mu}}-\Gamma_{\nu \lambda}^{\rho} a_{\rho \mu^{*}}=0, \\
& \nabla_{\nu^{*}} a_{\lambda \mu^{*}}=\partial_{\nu^{*}} a_{\lambda \mu^{*}}-\Gamma_{\nu^{*} \mu^{*}}^{\rho^{*}} a_{\lambda \rho^{*}}=0 .
\end{aligned}
$$

The $a_{\lambda \mu^{*}}$ is now a fundamental tensor and can be used to raise and lower in-

(7) The analytic group of transformations is given by $\xi^{\lambda^{\prime}}=\xi^{\lambda^{\prime}}\left(\xi^{\alpha}\right)$, conj.

(8) $[5$, vol. 2, p. 234]. 
dices through the $\nabla$ operator. If we define the contravariant fundamental tensor $a^{\sigma \cdot \lambda}$ by

$$
a^{0^{* \lambda}} a_{\infty 0^{*}}=A_{\mu}^{\lambda} \text {, conj., }
$$

where $A_{\mu}^{\lambda}$ is the unit affinor, then (2.17), (2.18) may be solved for the connection

$$
\begin{aligned}
\Gamma_{\mu \lambda}^{\alpha} & =a^{\sigma^{*} \alpha} \partial_{\mu} a_{\lambda \sigma^{*}}, \\
\Gamma_{\mu \lambda^{*}}^{\alpha^{*}} & =a^{\alpha^{* \sigma}} \partial_{\mu^{*} \cdot a_{\theta \lambda^{*}}}
\end{aligned}
$$

Finally, we introduce the torsion affinor

$$
\stackrel{S_{\mu \lambda}^{* \kappa}}{\ddot{*}}=(1 / 2)\left(\Gamma_{\mu \lambda}^{\alpha}-\Gamma_{\lambda \mu}^{\kappa}\right)=\Gamma_{[\mu \lambda]}^{\kappa} \text {, conj. }
$$

The sign [ ] means that the antisymmetric product of the enclosed indices is to be formed; the sign | | enclosing indices means that those indices are to be excluded in forming the antisymmetric product. When the torsion affinor can be written as

$$
S_{\mu \lambda}^{* \kappa}=A_{\left[\mu p_{\lambda]}\right.}^{\kappa}, \text { conj., }
$$

the unitary space $K_{n}$ is said to have a semi-symmetric connection.

3. Congruences in unitary $K_{n}$. Consider a vector field $u^{\lambda}\left(\xi^{\lambda}, \xi^{\lambda^{*}}\right)$ defined over the unitary $K_{n}$. The system of differential equations in the parameter $t$,

$$
d \xi^{\lambda} / u^{\lambda}=d t \text {, conj., }
$$

is said to define a congruence $\left(^{9}\right)$ of $\infty^{n-1}$ curves in the unitary $K_{n}$. We shall study the decomposition of the affinors $\nabla_{\alpha} u_{\lambda}, \nabla_{\alpha} \cdot u_{\lambda}$. Consider affinors $l_{\alpha \lambda}, l_{\alpha \cdot \lambda}$ which we define as the projections of $\nabla_{\alpha} u_{\lambda}, \nabla_{\alpha} \cdot u_{\lambda}$, respectively, upon the local $U_{n-1}$ which is unitary orthogonal( $\left.{ }^{10}\right)$ to $u_{\lambda}$. Hence, it follows that

$$
\begin{array}{rlrl}
u^{\alpha} l_{\alpha \lambda} & =0, & u^{\alpha^{*}} l_{\alpha^{*} \lambda}=0, \text { conj. }, \\
u^{\mu} l_{\alpha \mu}=0, & u^{\mu} l_{\alpha^{* \mu}}=0, \text { conj. }
\end{array}
$$

Furthermore, let $w_{\alpha}, z_{\alpha}, x_{\alpha}, y_{\alpha}$ be four arbitrary vectors in the above local $U_{n-1}$, that is,

$$
w_{\alpha} u^{\alpha}=z_{\alpha} u^{\alpha}=x_{\alpha} u^{\alpha}=y_{\alpha} u^{\alpha}=0, \text { conj. }
$$

We can now write( $\left.{ }^{11}\right)$

$$
\nabla_{\alpha} u_{\lambda}=l_{\alpha \lambda}+u_{\alpha} w_{\lambda}+z_{\alpha} u_{\lambda}+p u_{\alpha} u_{\lambda} \text {, conj., }
$$

(9) $[5$, vol. 2 , p. 27 , equation 5.1$]$.

(10) This local $U_{n-1}$ is determined by those vectors $u^{\lambda}$ (subscript $j=1, \cdots, n-1$ ) which are solutions of $u_{j}^{\lambda} u_{\lambda}=0$.

(Ii) $[5$, vol. 1, p. $19,8 \mathrm{k}]$. 


$$
\nabla_{\alpha} \cdot u_{\lambda}=l_{\alpha \cdot \lambda}+u_{\alpha \cdot x_{\lambda}}+y_{\alpha \cdot} \cdot u_{\lambda}+q^{*} u_{\alpha} \cdot u_{\lambda}, \text { conj., }
$$

where $p, q$ are scalars.

If the parameter $t$ in (3.1) is complex and the congruence curves are $U_{1}$ or if the parameter $t$ is real and hence the curves are $X_{1}$, then an analytic arc length parameter $s$ exists $\left({ }^{12}\right)$

$$
s=s(t) \text {, conj. }
$$

Now, if we replace the parameter $t$ by $s$ in the $\infty^{n-1}$ congruence curves, then the associated congruence vector $u^{\lambda}$ (we indicate the vector by the same symbol as before) is a unit vector, that is,

$$
u^{\lambda} u_{\lambda}=1 \text {, conj. }
$$

Because of (3.8), certain relations exist between the affinors in (3.5), (3.6). Before finding these relations, we formulate

Definition 1. (a) If the parameter $t$ in (3.1) is real, then the congruence defined by (3.1) will be said to be real. This congruence consists of $\infty^{n-1} X_{1}$ in $K_{n}$; (b) if the parameter $t$ is complex but the $\infty^{n-1}$ curves of the congruence are $U_{1}$, then we shall say that the congruence is complex Euclidean.

By covariant differentiation of (3.8), we obtain

$$
\left(\nabla_{\alpha} u_{\lambda}\right) u^{\lambda}=-\left(\nabla_{\alpha} u^{\lambda}\right) u_{\lambda} \text {, conj. }
$$

As a consequence of the equation

$$
u^{\lambda}=a^{\beta^{*} \lambda} u_{\beta^{*}}, \text { conj., }
$$

we find that the right-hand side of (3.9) can be expressed in terms of $\nabla_{\alpha} u_{\beta^{*}}$, that is,

$$
\left(\nabla_{\alpha} u_{\lambda}\right) u^{\lambda}=-\left(\nabla_{\alpha} u_{\beta}^{*}\right) u^{\beta^{*}}, \text { conj. }
$$

By use of (3.5), (3.6), the relation (3.11) can be shown to be equivalent to

$$
\begin{aligned}
z_{\alpha} & =-y_{\alpha}, \text { conj., } \\
p & =-q, \text { conj. }
\end{aligned}
$$

Conversely, if (3.12), (3.13) are valid, then the validity of (3.11), (3.9) follows. But (3.9) may be written in the form

$$
\nabla_{\alpha}\left(u_{\lambda} u^{\lambda}\right)=0, \text { conj. }
$$

Hence, it follows that

$$
u_{\lambda} u^{\lambda}=c \text {, conj., }
$$

where $c$ is some arbitrary constant in the unitary $K_{n}$. By use of (3.1), the

(12) $[1$, Theorems 3,4$]$. 
equation (3.15) becomes

$$
a_{\lambda \mu} d_{t} \xi^{\lambda} d_{i} \xi^{\mu^{*}}=c
$$

But this means that the curves of the congruence are $\infty^{n-1} U_{1}$ (for complex $t)\left({ }^{12}\right)$ or $\infty^{n-1} X_{1}$ (for real $t$ ). Hence, we have the theorem.

THEOREM 1. The necessary and sufficient conditions that the solutions $u_{\lambda}$ of (3.5), (3.6)-when they exist-should define either a real congruence or a complex Euclidean congruence is that $z_{\alpha}=-\dot{y}_{\alpha}, p=-q$.

4. Two systems of Pfaffians. Let us consider a general congruence vector $u_{\lambda}\left(\xi^{\lambda}, \xi^{\lambda^{*}}\right)$. In the first place, we associate with this vector a system of two Pfaffians

$$
\begin{aligned}
u_{\lambda} d \xi^{\lambda} & =0 \\
u_{\lambda} \cdot d \xi^{*} & =0
\end{aligned}
$$

Assuming that $u_{1}, u_{1} \cdot$ do not vanish over some region $D$ of the unitary $K_{n}$, we can rewrite the two previous equations in the form

$$
\begin{aligned}
& d \xi^{1}=-\sum^{\prime}\left(u_{\alpha} / u_{1}\right) d \xi^{\alpha}, \quad \alpha=2, \cdots, n, \\
& d \xi^{1^{*}}=-\sum^{\prime}\left(u_{\alpha^{*}} / u_{1^{*}}\right) d \xi^{\alpha^{*}}
\end{aligned}
$$

where $\sum^{\prime}$ denotes summation over all repeated indices with the exception of the index 1 . If the integrability conditions of this system are satisfied, we can solve( $\left.{ }^{13}\right)$ for $\xi^{1}, \xi^{1^{*}}$

$$
\begin{aligned}
& \xi^{1}=\xi^{1}\left(\xi^{\alpha}, \underset{0}{\alpha^{*}}, \underset{0}{\xi^{1}}, \xi^{*^{*}}\right), \quad \alpha=2, \cdots, n, \\
& \xi^{*^{*}}=\xi^{1^{*}}\left(\xi^{\alpha}, \underset{0}{\xi^{*}}, \underset{0}{\xi^{1}}, \xi^{1^{*}}\right)
\end{aligned}
$$

where: (1) $\xi^{1}, \xi^{1^{*}}$ (subindex 0$)$ are arbitrary constants; (2) $\xi^{\alpha}, \xi^{\alpha^{*}}(\alpha=2, \cdots, n)$ are the independent variables; ( 3$) \xi^{1}, \xi^{*}$ are the dependent variables. By solving for $\xi^{1}, \xi^{\bullet}$ (subindex 0 ), we obtain the two independent integrals of (4.1), (4.2),

$$
\begin{aligned}
& \xi^{1}=f\left(\xi^{\lambda}, \xi^{\lambda^{*}}\right), \\
& 0 \\
& \xi^{1^{*}}=g\left(\xi^{\lambda}, \xi^{\lambda^{*}}\right) .
\end{aligned}
$$

We now prove

LEMMA 1. The two independent integrals $f, g$ are conjugate functions.

The equations (4.5), (4.6) become identities when the variables $\xi^{\lambda}, \xi^{\lambda^{*}}$ $(\lambda=1,2, \cdots, n)$ are replaced by the arbitrarily assigned constants $\xi^{\lambda}, \xi^{\lambda^{*}}$

(13) $[4$, p. 49]. 
(subindex 0). Hence (4.7), (4.8) become identities when the same substitution is made. That is, the functions $f$ and $g$ reduce to conjugate quantities $\xi^{1}, \xi^{1^{*}}$ (subindex 0 ) when the $\xi^{\lambda}, \xi^{\lambda^{*}}(\lambda=1,2, \cdots, n)$ are assigned arbitrary values. Thus, $f$ and $g$ are conjugate functions.

Since the quantity $\xi^{*}$ (subindex 0 ) is known when the quantity $\xi^{1}$ (subindex 0 ) has been assigned some arbitrary value, we shall say that (4.7), (4.8) determine $\infty^{1}$ integrals of (4.1), (4.2). We prove

Lemma 2. The $\infty^{1}$ integrals of (4.1), (4.2) determine $\infty^{1}$ unitary hypersurfaces $K_{n-1}$ in $K_{n}$.

Let us denote $\xi^{\alpha}, \xi^{\alpha^{*}}(\alpha=2, \cdots, n)$ by $u^{a}, u^{a^{*}}(a=1, \cdots, n-1)$, respectively; these $u^{a}, u^{a^{*}}$ will serve as the hypersurface intrinsic parameters. Thus, (4.7), (4.8) or (4.5), (4.6) can be written as

$$
\xi^{1}=\xi^{1}\left(u^{a}, u^{a^{*}}, \xi_{0}^{1}, \xi^{1^{*}}\right) \text {, conj. }
$$

If the $u^{a^{*}}$ actually occur in the right-hand side of (4.9), then these equations define $\infty^{1}$ semi-analytic $\left({ }^{2}\right)$ hypersurfaces $X_{n-1}$ in the unitary $K_{n}$. We shall show that these $u^{a^{*}}$ do not occur. By forming the total differential of (4.9), we obtain

$$
d \xi^{1}=d u^{a} \partial_{a} \xi^{1}+d u^{a^{*}} \partial_{a} \xi^{1}, \text { conj. }
$$

Since the $d u^{a}, d u^{\alpha^{*}}$ are equal to the differentials $d \xi^{\alpha}, d \xi^{\alpha^{*}}(\alpha=2, \cdots, n)$ of the independent variables $\xi^{\alpha}, \xi^{\alpha^{*}}$, we find by comparing (4.10) and its conjugate with (4.3), (4.4) that

$$
\partial_{a} \cdot \xi^{1}=0, \text { conj. }
$$

Hence (4.9) may be written in the form

$$
\xi^{1}=\xi^{1}\left(u^{a}, \underset{0}{\xi^{1}}, \xi^{1 *}\right), \text { conj. }
$$

The equations (4.12) determine $\infty^{1}$ analytic hypersurfaces $X_{n-1}$ in the unitary $K_{n}$. Such analytic hypersurfaces are always unitary(14) $K_{n-1}$. Hence our lemma is proved.

In view of the fact that (4.1), (4.2) are unitary orthogonality relations, we state

DEFINITION 2. The integrals $f=$ const. and $f^{*}=$ const. of (4.1), (4.2) will be said to define $\infty^{1}$ hypersurfaces in the unitary $K_{n}$ such that the hypersurfaces are completely unitary orthogonal to the congruence vector $u_{\lambda}$.

We may restate Lemma 2 in terms of Definition 2 as follows:

(14) $[5$, vol. 2 , p. 245$]$. 
LEMMA 3. The $\infty^{1}$ hypersurfaces which are completely unitary orthogonal to the congruence vector $u_{\lambda}$ are $\infty^{1}$ unitary $K_{n-1}$ in the unitary $K_{n}$.

Let us now consider the single Pfaffian

$$
u_{\lambda} d \xi^{\lambda}+u_{\lambda} \cdot d \xi^{\lambda}=0 .
$$

By assuming that $u_{1}$ does not vanish over some domain $D$ of the unitary $K_{n}$, we can construct a theory of this Pfaffian in which (4.3) is replaced by

$$
d \xi^{1}=-\sum^{\prime}\left(u_{\alpha} / u_{1}\right) d \xi^{\alpha}-\left(u_{\lambda} \cdot u_{1}\right) d \xi^{\star *}, \quad \alpha=2, \cdots, n ; \lambda=1, \cdots, n .
$$

The equation (4.5) becomes

$$
\xi^{1}=\xi^{1}\left(\xi^{\alpha}, \xi^{\lambda *}, \xi_{0}^{1}\right)
$$

Furthermore, (4.13) has only one independent integral

$$
\xi^{1}=f\left(\xi^{\lambda}, \xi^{\lambda *}\right)
$$

The equation corresponding to (4.4) is identical with (4.14); the equation corresponding to (4.6) is the conjugate of (4.15). Finally, the equation corresponding to (4.8) is equivalent to the conjugate of (4.16). However, this last equation is trivial since if $f^{*}=$ const. is an integral of (4.13), then $f=F\left(f^{*}\right)$.

We now define a new term.

Definition 3. The integral $f=$ const. of (4.13) will be said to define $\infty^{1}$ hypersurfaces $X_{n-1}$ in the unitary $K_{n}$ which are semi-unitary orthogonal to the congruence $u_{\lambda}$.

These semi-unitary orthogonal hypersurfaces can be characterized by their parameter representation. We prove

LEMMA 4. The $\infty^{1}$ semi-unitary orthogonal hypersurfaces to the congruence $u_{\lambda}$ cannot possess an analytic parameter representation of rank $(n-1)$. That is, these $X_{n-1}$ are not unitary $K_{n-1}$.

Let us assume the contrary, namely, that these hypersurfaces possess an analytic parameter representation.

$$
\xi^{\lambda}=\xi^{\lambda}\left(u^{a}\right), \quad \lambda=1, \cdots, n ; a=1, \cdots, n-1, \text { conj. }
$$

Since the rank of $(4.17)$ is $(n-1)$, we can solve for the $(n-1) u^{a}$ in terms of $(n-1)$ of the $\xi^{\alpha}$ (say, $\left.\alpha=2, \cdots, n\right)$,

$$
u^{a}=u^{a}\left(\xi^{\alpha}\right) \text {, conj. }
$$

Substituting (4.18) into the first equation of (4.17), we obtain

$$
\xi^{1}=\xi^{1}\left(\xi^{\alpha}\right) \text {, conj. }
$$

Forming the total differential of (4.19), we find 


$$
d \xi^{1}=d \xi^{\alpha} \partial_{\alpha} \xi^{1},
$$
$\alpha=2, \cdots, n$, conj.

Remembering that $\xi^{\alpha}, \xi^{\alpha^{*}}, \xi^{*}(\alpha=2, \cdots, n)$ are independent variables and comparing with (4.14), we obtain

$$
u_{\lambda^{*}}=0, \quad \lambda=1, \cdots, n, \text { conj. }
$$

Hence the congruence vector vanishes. Thus, the assumption (4.17) is false for a non-vanishing congruence; our lemma is proved. Our lemma implies that if the class of semi-unitary orthogonal hypersurfaces can be parameterized, the parameterization is semi-analytic (see 4.9).

Another relation exists between the $\infty^{1}$ completely unitary orthogonal hypersurfaces and the $\infty^{1}$ semi-unitary orthogonal hypersurfaces. Let us consider the systems of partial differential equations associated with (4.1), (4.2) and (4.13). The system associated with (4.1), (4.2) is

$$
\begin{aligned}
\partial_{\alpha} f-\left(u_{\alpha} / u_{1}\right) \partial_{1} f=0, & \alpha=2, \cdots, n, \\
\partial_{\alpha^{*}} f-\left(u_{\alpha^{*}} / u_{1^{*}}\right) \partial_{1^{*}} f & =0 .
\end{aligned}
$$

The system associated with (4.13) is composed of (4.22), (4.23) plus the additional equation

$$
\partial_{1} \vee f-\left(u_{1} * / u_{1}\right) \partial_{1} f=0 .
$$

If $\partial_{1} f, \partial_{1} * f$ do not vanish over the domain $D$ in which $u_{1}, u_{1^{*}}$ do not vanish, then the non-vanishing scalars $\rho, \gamma$ exist such that (4.22), (4.23)-hence (4.1), (4.2)-are equivalent to

$$
\begin{gathered}
u_{\lambda}=\rho \nabla_{\lambda} f, \\
u_{\lambda^{*}}=\gamma \nabla_{\lambda} * f .
\end{gathered}
$$

The equation (4.13) is equivalent to (4.25), (4.26) plus the additional equation (4.24). However, the latter implies

$$
\gamma=\rho .
$$

Before proceeding to enumerate these new results, we note that if $f=$ const. is a solution of (4.22) through (4.24), then $f^{*}=$ const. is a solution of the same equations (see the discussion following 4.16). Hence the word "conjugate" can be written after equations (4.25) through (4.27).

LEMMA 5. The solutions $f=$ const. (and its conjugate) of (4.25), (4.26) where $\rho \neq \gamma$ determine $\infty^{1}$ completely unitary orthogonal hypersurfaces (unitary $K_{n-1}$ ) to the congruence. The solution $f=$ const. of (4.25), (4.26) where $\rho=\gamma$ determine $\infty^{1}$ semi-unitary orthogonal hypersurfaces $X_{n-1}$ to the congruence.

5. Congruences completely unitary orthogonal to $\infty^{1} K_{n-1}$ in $K_{n}$. We consider congruences which are completely unitary orthogonal to $\infty^{1}$ unitary 
$K_{n-1}$ in unitary $K_{n}$. The integrability conditions(15) of (4.1), (4.2) are

$$
\begin{gathered}
u_{\lambda} \partial_{[\beta} u_{\alpha]}+u_{\alpha} \partial_{[\lambda} u_{\beta]}+u_{\beta} \partial_{[\alpha} u_{\lambda]}=0, \text { conj. } \\
u_{\beta} \partial_{\alpha} u_{\lambda}-u_{\lambda} \partial_{\alpha} * u_{\beta}=0, \text { conj. }
\end{gathered}
$$

By use of (2.11), (2.22), we find

$$
\begin{aligned}
\partial_{[\beta} u_{\alpha]} & =\nabla_{[\beta} u_{\alpha]}+\stackrel{S_{\beta \alpha}^{* \gamma} u_{\gamma}, \text { conj. }}{\partial_{\alpha} \cdot u_{\lambda}}=\nabla_{\alpha} \cdot u_{\lambda}, \text { conj. }
\end{aligned}
$$

Thus (5.1), (5.2) become

$$
\begin{aligned}
u_{\left[\lambda \nabla_{\beta}\right.} u_{\alpha]} & =-u_{[\lambda} \cdot \stackrel{S_{\beta \alpha]}^{* \gamma}}{u_{\gamma}}, \text { conj., } \\
u_{\left[\beta \nabla\left|\alpha^{*}\right| u_{\lambda]}\right.} & =0 \text {, conj. }
\end{aligned}
$$

By transvecting (5.2) or (5.6) with $u^{\lambda}$, we find

$$
\nabla_{\alpha^{*}} u_{\beta}=u_{\beta}\left(\nabla_{\alpha^{*}} u_{\lambda}\right) u^{\lambda} \text {, conj. }
$$

Replacing the right-hand side by (3.6), we obtain

$$
\nabla_{\alpha} \cdot u_{\beta}=y_{\alpha} \cdot u_{\beta}+q^{*} u_{\alpha} \cdot u_{\beta}, \text { conj. }
$$

Hence, upon comparing (5.8) with (3.6), we find that

$$
l_{\alpha^{* \lambda}}=0, \quad x_{\lambda}=0, \text { conj., }
$$

is a consequence of the integrability conditions (5.6). We now study the meaning of the integrability conditions (5.5). Let us assume that the connection of $K_{n}$ is semi-symmetric (see 2.23 ). Then the equation (5.5) reduces to

$$
u_{[\lambda} l_{\beta \alpha]}=0 \text {, conj., }
$$

in consequence of (3.5) and (5.5). By transvecting (5.10) with $u^{\lambda}$ and using (3.2), (3.3), this equation becomes

$$
l_{[\beta \alpha]}=0, \text { conj. }
$$

Conversely, if (5.9) and (5.11) are valid and if the connection of $K_{n}$ is semisymmetric, then the expressions (3.5), (3.6) satisfy the integrability conditions (5.5), (5.6). This leads us to

Theorem 2. Consider a unitary space $K_{n}$ with semi-symmetric connection and such that the solutions $u_{\lambda}$ of (3.5), (3.6) exist then if and only if: (1) $l_{\alpha \beta}$ is symmetric; (2) $l_{\alpha * \beta}, x_{\lambda}$ vanish, does the vector $u_{\lambda}$ define a congruence which is completely unitary orthogonal to $\infty^{1}$ hypersurfaces in the unitary $K_{n}$.

(16) $\left[4\right.$, p. 29, equation 23]. Since the $u_{\lambda}, u_{\lambda^{*}}$ in (4.1), (4.2) are functions of $\xi^{\mu}$ and $\xi^{\mu *}$, the complete Pfaffians in (4.1), (4.2) can be written as $w_{\lambda} d \xi^{\lambda^{*}}+u_{\lambda} d \xi^{\lambda}=0, u_{\lambda^{*}} d \xi^{\lambda^{*}}+w_{\lambda} d \xi^{\lambda}=0$, where $w_{\lambda}, w_{\lambda} *=0$. If one writes out the equation 23, p. 29 of $[4]$, then for unstarred variables (or inindices) the equation (5.1) results; if one of the starred variables (or indices) is used then equation (5.2) results. 
Let us now restrict ourselves to real congruences (see Definition 1 (a)). By introducing the Frenet formulas $\left({ }^{16}\right)$ for the $\infty^{n-1}$ curves $X_{1}$ of the congruence, we can determine the meaning of the vector $w_{\alpha}$ in (3.5). From the Frenet formulas, it follows that

$$
u^{\alpha} \nabla_{\alpha} u_{\lambda}+u^{\alpha^{*}} \nabla_{\alpha^{*}} u_{\lambda}=\underset{00}{k} u_{\lambda}+\underset{011}{k} u_{\lambda} \text {, conj., }
$$

where $k$ (subindex 00,01 ) are curvatures and $u_{\lambda}$ (subindex 1 ) is the first normal of each $X_{1}$ in the unitary $K_{n}$. By use of (3.5), (3.6), (3.13), we find

$$
u^{\alpha} \nabla_{\alpha} u_{\lambda}+u^{\alpha^{*}} \nabla_{\alpha} u_{\lambda}=w_{\lambda}+x_{\lambda}+\left(p-p^{*}\right) u_{\lambda} \text {, conj. }
$$

By comparison of (5.12), (5.13), we obtain

$$
\begin{aligned}
w_{\lambda}+x_{\lambda}=\underset{\substack{k 1 \\
01}}{k} u_{\lambda}, \text { conj., } \\
p-p^{*}=\underset{\substack{00 \\
k}}{k} \text { conj. }
\end{aligned}
$$

If we require that the congruence shall be completely unitary orthogonal to $\infty^{1}$ hypersurfaces in the unitary $K_{n}$, then it follows from Theorem 2 that the vector $x_{\lambda}$ vanishes. Hence, we obtain the result

THEOREM 3. If the congruence is real and completely unitary orthogonal to $\infty^{1}$ hypersurfaces in the unitary $K_{n}$, then (1) the vector $w_{\lambda}$ lies along the first normal to any $X_{1}$ of the congruence; (2) the magnitude of $w_{\lambda}$ is equal to the $(0,1)$ curvature of $X_{1} ;(3)$ the imaginary part of the scalar $p$ is one-half the $(0,0)$ curvature of $X_{1}$.

Again, let us consider the case where the congruence is completely unitary. orthogonal to $\infty^{1}$ hypersurfaces in the unitary $K_{n}$ and where the congruence is either real or complex Euclidean. By means of Theorems 1 and 2, we may write (3.5), (3.6) in the form

$$
\begin{aligned}
\nabla_{\alpha} u_{\lambda} & =l_{\alpha \lambda}+u_{\alpha} w_{\lambda}+h_{\alpha} \dot{u_{\lambda}}, \text { conj., } \\
\nabla_{\alpha} u_{\lambda} & =-h_{\alpha^{*}} u_{\lambda}, \text { conj., }
\end{aligned}
$$

where

$$
h_{\alpha}=z_{\alpha}+p u_{\alpha}, \text { conj. }
$$

From (5.17), we see that if $\nabla_{\alpha} \cdot u_{\lambda}$ vanishes, then $h_{\alpha}$ vanishes. Hence from (5.18), we find that the vector $z_{\alpha}$ and the scalar $p$ vanish. By (5.15), this last result means that the curvature $k$ (subindex 00 ) of a real congruence vanishes. Furthermore, the equations (5.16) and (5.11) furnish the result

$$
\nabla_{\alpha} u_{\lambda}=l_{\alpha \lambda}+u_{\alpha} w_{\lambda} \text {, conj. }
$$

\footnotetext{
(16) [2, equation 3.23].
} 
The equation (5.19) is the condition $\left({ }^{3}\right)$ satisfied by a congruence of curves $V_{1}$ which are orthogonal to $\infty^{1}$ hypersurfaces in a Riemannian space of $n$ dimensions $V_{n}$. Hence, we have the result

THEOREM 4. If the vector $u_{\lambda}$ determines a congruence which is: (1) completely unitary orthogonal to $\infty^{1}$ hypersurfaces in the unitary $K_{n}$; (2) the vector $u_{\lambda}$ is analytic; (3) the congruence is either real or complex Euclidean, then the conditions satisfied by the congruence vector $u_{\lambda}$ in the unitary $K_{n}$ are identical with those satisfied by the congruence vector $u_{\lambda}$ which is orthogonal to $\infty^{1}$ hypersurfaces in $V_{n}$. If the condition (3) is replaced by the stronger requirement that the congruence is real, then a further conclusion follows. Namely, the curvature $k$ (subindex 00) vanishes.

6. Congruences semi-unitary orthogonal to $\infty^{1} X_{n-1}$ in $K_{n}$. If the congruence is semi-unitary orthogonal to $\infty^{1}$. hypersurfaces in the unitary $K_{n}$, then the integrability conditions of (4.13) must be satisfied. These are given by

$$
\begin{aligned}
u_{\lambda} \partial_{[\beta} u_{\alpha]}+u_{\alpha} \partial_{[\lambda} u_{\beta]}+u_{\beta} \partial_{[\alpha} u_{\lambda]}=0, \text { conj., } \\
u_{\lambda} \partial_{\left[\beta^{*} u_{\alpha]}+u_{\alpha} \partial_{[\lambda} u_{\beta}{ }^{*}\right]}+u_{\beta} \partial_{[\alpha} u_{\lambda]}=0, \text { conj. }
\end{aligned}
$$

By use of (5.3), (5.4), the equations (6.1), (6.2) become

$$
\begin{aligned}
u_{\left[\lambda \nabla_{\beta}\right.} u_{\alpha]} & =-u_{[\lambda} \stackrel{\cdot * \gamma}{S_{\beta \alpha]}} u_{\gamma}, \text { conj., } \\
u_{\left[\lambda \nabla_{\beta} * u_{\alpha]}\right.} & =-(1 / 3) u_{\beta} * S_{\alpha \lambda} \cdot u_{\gamma}, \text { conj. }
\end{aligned}
$$

Let us assume that the connection of $K_{n}$ is semi-symmetric (see 2.23). Then, the right-hand side of (6.3) vanishes and the right-hand side of (6.4) becomes $\left(-(1 / 3) u_{\beta} * u_{\left[\alpha p_{\lambda]}\right]}\right.$. Upon substituting (3.5) in to (6.3), we obtain

$$
u_{[\lambda} l_{\beta \alpha]}=0 \text {, conj. }
$$

Transvecting with $u^{\lambda}$ and using (3.2), (3.3), we conclude that

$$
l_{[\beta \alpha]}=0 \text {, conj. }
$$

Conversely, if (6.6) is valid, then (3.5) satisfies (6.3). We next study the consequences of (6.4). By transvecting (6.4) with $u^{\alpha}, u^{\beta^{*}}$, we obtain

$$
\begin{aligned}
& u_{\lambda} u^{\alpha} \nabla_{\left[\beta^{*}\right.} u_{\alpha]}+\nabla_{[\lambda} u_{\left.\beta^{*}\right]}+u_{\beta^{*}} u^{\alpha} \nabla_{[\alpha} u_{\lambda]}=-u_{\beta^{*}} u^{\alpha} u_{[\alpha} p_{\lambda]}, \text { conj., } \\
& u_{\lambda} u^{\beta^{*}} \nabla_{\left[\beta^{*}\right.} u_{\alpha]}+u_{\alpha} u^{\beta^{*}} \nabla_{[\lambda} u_{\left.\beta^{*}\right]}+\nabla_{[\alpha} u_{\lambda]}=-u_{[\alpha} p_{\lambda]}, \text { conj. }
\end{aligned}
$$

Due to the symmetry of (6.4), no additional relations are obtained by further transvection with $u^{\lambda}$. With the aid of (3.5), (3.6), (6.6), the two previous equations become

$$
\begin{aligned}
& u_{\lambda} u^{\alpha}\left(u_{\left[\beta^{*}\right.} x_{\alpha]}+y_{\left[\beta^{*}\right.} u_{\alpha]}\right)+l_{\left[\lambda \beta^{*}\right]}+u_{[\lambda} x_{\left.\beta^{*}\right]}+y_{[\lambda} u_{\left.\beta^{*}\right]} \\
&+u_{\beta^{*}} u^{\alpha}\left(u_{[\alpha} w_{\lambda]}+z_{[\alpha} u_{\lambda]}\right)=-u_{\beta} u^{\alpha} u_{[\alpha} p_{\lambda]}, \text { conj., } \\
& u_{\lambda} u^{\beta^{*}}\left(u_{\left[\beta^{*} x_{\alpha]}\right.}+y_{\left[\beta^{*} u_{\alpha]}\right)}+u_{\alpha} u^{\beta^{*}}\left(u_{[\lambda} x_{\left.\beta^{*}\right]}+y_{[\lambda} u_{\left.\beta^{*}\right]}\right)\right. \\
&+\left(u_{[\alpha} w_{\lambda]}+z_{[\alpha} u_{\lambda]}\right)=-u_{\left[\alpha p_{\lambda]},\right. \text { conj. }}
\end{aligned}
$$


Simplifying with the aid of (3.4), we find

(6.11) $2 l_{\left[\beta^{*}\right]}-u_{\beta^{*}}\left(x_{\lambda}+z_{\lambda}-y_{\lambda}-w_{\lambda}\right)=-u_{\beta^{*}}\left[p_{\lambda}-u_{\lambda}\left(p_{\alpha} u^{\alpha}\right)\right]$, conj.,

(6.12) $-u_{\alpha}\left(x_{\lambda}+z_{\lambda}-y_{\lambda}-w_{\lambda}-p_{\lambda}\right)+u_{\lambda}\left(x_{\alpha}+z_{\alpha}-y_{\alpha}-w_{\alpha}-p_{\alpha}\right)=0$, conj.

Transvecting (6.11) with $u^{\beta^{*}}$ and simplifying with (3.2), (3.3), we obtain

$$
x_{\lambda}+z_{\lambda}-y_{\lambda}-w_{\lambda}=p_{\lambda}-u_{\lambda}\left(p_{\alpha} u^{\alpha}\right) \text {, conj. }
$$

Substituting (6.13) into (6.11), we find

$$
l_{\left[\lambda \beta^{*}\right]}=0 \text {, conj. }
$$

By substituting (6.13) into (6.12), we find that the latter equation is identically satisfied. Thus (6.13), (6.14) are the only equations obtained by transvecting (6.4) with $u^{\lambda}$. Conversely, by expanding (6.4) and using (3.5), (3.6), we find that in virtue of (6.6), (6.14), (6.13), the equation (6.4) is identically satisfied. This leads us to a theorem which is similar to Theorem 2 ,

THEOREM 5. Consider a unitary space $K_{n}$ with semi-symmetric connection and such that the solutions $u_{\lambda}$ of (3.5), (3.6) exist, then if and only if: (1) $l_{\alpha \beta}, l_{\alpha^{* \beta}}$ are symmetric; (2) $x_{\lambda}+z_{\lambda}-y_{\lambda}-w_{\lambda}=p_{\lambda}-u_{\lambda}\left(p_{\alpha} u^{\alpha}\right)$, does the vector $u_{\lambda}$ define a congruence which is semi-unitary orthogonal to $\infty^{1}$ hypersurfaces in the unitary $K_{n}$.

By use of Lemma 5, we can obtain some further properties of the semiunitary congruences. From (4.25), (4.26), (4.27) it follows that for congruences which are semi-unitary orthogonal to $\infty^{1}$ hypersurfaces in the uni$\operatorname{tary} K_{n}$

$$
\begin{aligned}
u_{\lambda} & =\rho \nabla_{\lambda} f, \text { conj. }, \\
u_{\lambda^{*}} & =\rho \nabla_{\lambda^{*}} f, \text { conj. }
\end{aligned}
$$

By covariant differentiation of (6.15), (6.16), we find

$$
\begin{aligned}
\nabla_{\alpha} u_{\lambda} & =\rho \nabla_{\alpha} \nabla_{\lambda} f+\left(\nabla_{\lambda} f\right)\left(\nabla_{\alpha} \rho\right), \text { conj., } \\
\nabla_{\alpha} u_{\lambda^{*}} & =\rho \nabla_{\alpha} \nabla_{\lambda} \cdot f+\left(\nabla_{\lambda^{*}} f\right)\left(\nabla_{\alpha} \rho\right), \text { conj. }
\end{aligned}
$$

From the relations (2.11), (2.22), we see that

$$
\begin{aligned}
\nabla_{\alpha} \nabla_{\lambda} f & =\nabla_{\lambda} \nabla_{\alpha} f+2 S_{\lambda \alpha}^{\cdot \cdot \gamma} \nabla f, \text { conj., } \\
\nabla_{\alpha} \nabla_{\lambda} \cdot f & =\nabla_{\lambda} \cdot \nabla_{\alpha} f, \text { conj. }
\end{aligned}
$$

Substituting the last two equations into (6.17), (6.18), the latter become

$$
\begin{aligned}
& \nabla_{\alpha} u_{\lambda}=\rho \nabla_{\lambda} \nabla_{\alpha} f+2 \rho S_{\lambda \alpha}^{* \gamma} \nabla_{\gamma} f+\left(\nabla_{\lambda} f\right)\left(\nabla_{\alpha} \rho\right), \text { conj., } \\
& \nabla_{\alpha} u_{\lambda^{*}}=\rho \nabla_{\lambda} \cdot \nabla_{\alpha} f+\left(\nabla_{\lambda} * f\right)\left(\nabla_{\alpha} \rho\right) \text { conj., }
\end{aligned}
$$

Simplifying (6.21), (6.22) by use of the equations (6.15) through (6.18), we 
obtain

$$
\begin{aligned}
\nabla_{\alpha} u_{\lambda} & =\nabla_{\lambda} u_{\alpha}+2 S_{\lambda \alpha}^{\cdot \gamma} u_{\gamma}+2 \rho^{-1} u_{[\lambda} \nabla_{\alpha]} \rho, \text { conj. } \\
\nabla_{\alpha} u_{\lambda^{*}} & =\nabla_{\lambda} \cdot u_{\alpha}+2 \rho^{-1} u_{[\lambda} \cdot \Delta_{\alpha]} \rho, \text { conj. }
\end{aligned}
$$

By use of (3.5), (3.6), (6.6), (6.14), the above two equations become

$$
\begin{aligned}
u_{[\alpha} w_{\lambda]}+z_{[\alpha} u_{\lambda]} & =S_{\lambda \alpha}^{\cdot \gamma} u_{\gamma}+\rho^{-1} u_{\left[\lambda \nabla_{\alpha]} \rho,\right. \text { conj. }}, \\
u_{[\alpha} x_{\left.\lambda^{*}\right]}+y_{[\alpha} u_{\left.\lambda^{*}\right]} & =\rho^{-1} u_{\left[\lambda \cdot \nabla_{\alpha]} \rho,\right. \text { conj. }}
\end{aligned}
$$

Let us assume that the connection of $K_{n}$ is semi-symmetric. Transvecting the previous two equations with $u^{\alpha}$, we obtain

$$
\begin{aligned}
w_{\lambda}-z_{\lambda} & =-p_{\lambda}+u_{\lambda}\left(p_{\alpha} u^{\alpha}\right)-\nabla_{\lambda} \ln \rho+u_{\lambda}\left(u^{\alpha} \nabla_{\alpha} \ln \rho\right), \text { conj., } \\
x_{\lambda^{*}}-y_{\lambda^{*}} & =-\nabla_{\lambda^{*}} \ln \rho+u_{\lambda^{*}}\left(u^{\alpha} \nabla_{\alpha} \ln \rho\right), \text { conj. }
\end{aligned}
$$

We are now in a position to prove

THEOREM 6. If: (1) the connection of the unitary $K_{n}$ is semi-symmetric; (2) the congruence is semi-unitary orthogonal to $\infty^{1}$ hypersurfaces in $K_{n}$; (3) the congruence is real or complex Euclidean; (4) $w_{\lambda}=z_{\lambda}, x_{\lambda}=y_{\lambda}$, then every two hypersurfaces $X_{n-1}$ intercept equal arc segments on all curves of the congruence.

From condition (4) of our theorem, that is,

$$
w_{\lambda}=z_{\lambda}, \quad x_{\lambda}=y_{\lambda}, \text { conj., }
$$

it follows by use of (6.13) that

$$
p_{\lambda}=\left(p_{\alpha} u^{\alpha}\right) u_{\lambda}, \text { conj. }
$$

Substituting (6.29), (6.30) into (6.27), (6.28), we find

$$
\begin{aligned}
u_{\lambda} & =\theta \nabla_{\lambda} \rho, \text { conj., } \\
u_{\lambda^{*}} & =\theta \nabla_{\lambda} \rho, \text { conj., }
\end{aligned}
$$

where $\theta$ is some function of $\xi^{\lambda}, \xi^{\lambda^{*}}$. Thus $\rho$ is an integral of the system (4.22) through (4.24). Since that system has only one independent integral, namely, $f\left(\xi^{\lambda}, \xi^{\lambda^{*}}\right)$, it follows that

$$
\rho=F(f), \text { conj., }
$$

where $F(f)$ is some arbitrary function of $f$. From (6.33), (6.15), (6.16), we find (6.34)

$$
F(f) d f=u_{\lambda} d \xi^{\lambda}+u_{\lambda} \cdot d \xi^{\lambda^{*}},
$$

for arbitrary $d \xi^{\lambda}, d \xi^{\lambda^{*}}$. Now let us consider the vector $\left(d \xi^{\lambda}, d \xi^{\lambda^{*}}\right)$ as in the direction of $u^{\lambda}$. By multiplying and dividing the right-hand side of (6.34) by $d s$ (the element of arc length along a curve of the congruence), we obtain

$$
F(f) d f=2 d s .
$$


Integrating (6.35) between $f=c_{0}, f=c_{1}$, we find

$$
2\left(s-s_{0}\right)=\int_{c_{0}}^{c_{1}} F(f) d f .
$$

The fact that the right-hand side of (6.36) is independent of any particular curve of the congruence proves our theorem.

We can obtain the geometric meaning of the essential condition (4) in Theorem 6 by limiting ourselves to real congruences. We prove

LEMMA 6. Consider a real congruence which is semi-unitary orthogonal to $\infty^{1}$ hypersurfaces in a unitary $K_{n}$ with semi-symmetric connection, then if and only if: (1) the (01) curvature of each $X_{1}$ vanishes; (2) $p_{\lambda}=\left(p_{\alpha} u^{\alpha}\right) u_{\lambda}$, are the equations (6.29) valid.

First, we show the sufficiency of our conditions. From the first condition, we have

$$
\underset{01}{k}=0 \text {, conj. }
$$

Hence from (5.14), it follows that

$$
w_{\lambda}+x_{\lambda}=0 \text {, conj. }
$$

Since the congruence is real, the equation (3.12), is valid, that is,

$$
z_{\lambda}=-y_{\lambda} \text {, conj. }
$$

By use of the second condition and (6.13), we obtain

$$
x_{\lambda}+z_{\lambda}-y_{\lambda}-w_{\lambda}=0 \text {, conj. }
$$

By substituting (6.38), (6.39) into (6.40), we obtain the equations (6.29).

Conversely, from (6.29) and the fact that the congruence is real, it follows that the conditions (1), (2) of our theorem are satisfied. Thus, from (6.29) and (6.13), we obtain

$$
p_{\lambda}=\left(p_{\alpha} u^{\alpha}\right) u_{\lambda} \text {, conj. }
$$

Furthermore, by use of (6.39), (6.29) and (5.14), we find that $k$ (subindex 01) vanishes.

We now translate Theorem 6 into terms connected with a real congruence of geodesic curves. Our result is

THEOREM 7. If the curves $X_{1}$ of a real congruence in a unitary space $K_{n}$ with semi-symmetric connection satisfy the conditions: (1) the congruence is semiunitary orthogonal to $\infty^{1}$ hypersurfaces in $K_{n}$; (2) the $\infty^{n-1} X_{1}$ are geodesic; and either (3) the curvature $k$ (subindex 01) vanishes; or (4) $p_{\lambda}=\left(p_{\alpha} u^{\alpha}\right) u_{\lambda}$, then every two hypersurfaces intercept equal arc segments on all $X_{1}$ of the congruences. 
It has been shown $\left({ }^{17}\right)$ that an $X_{1}$ in a unitary $K_{n}$ with semi-symmetry connection is geodesic if and only if

$$
\begin{aligned}
p_{\lambda} & =\left(p_{\alpha} u^{\alpha}\right) u_{\lambda}-\underset{011}{k} u_{\lambda}, \text { conj., } \\
k & =0, \text { conj. }
\end{aligned}
$$

The condition (6.43) is of no use to us. From (6.42), we note that if condition (3) of our theorem is valid, then the condition (4) is necessarily satisfied, and conversely. From Lemma 6, it follows that (6.29) is valid. Hence, Lemma 6 leads to the desired conclusion.

If in particular, the space $K_{n}$ has a symmetric connection, then

$$
p_{\lambda}=0 \text {, conj. }
$$

Thus the conditions (3), (4) of Theorem 7 are satisfied. Theorem 7 becomes analogous to a theorem in Riemannian space $\left({ }^{4}\right)$.

\section{REFERENCES}

1. N. Coburn, Unitary curves in unitary space, Revista de la Universidad Nacional de Tucuman vol. 2 (1941) pp. 159-167.

2. - Frenet formulas for curves in unitary space, Journal of Mathematics and Physics, Massachusetts Institute of Technology vol. 21 (1942) pp. 10-18.

3. - Semi-analytic unitary subspaces of unitary space, Amer. J. Math. vol. 64 (1942) pp. 714-724.

4. T. Levi-Civita, The absolute differential calculus, London, 1929.

5. J. A. Schouten and D. J. Struik, Einfuhrung in die Neueren Methoden der Differentialgeometrie, Groningen, vol. 1, 1935; vol. 2, 1938.

UNIVERSITY OF TEXAS, Austin, Texas

(17) $[2$, Theorem 4$]$. 